\title{
First-Level Transitivity Rule Method for Filling in Incomplete Pair-Wise Comparison Matrices in the Analytic Hierarchy Process
}

\author{
Bojan Srdjevic*, Zorica Srdjevic and Bosko Blagojevic \\ Department of Water Management, Faculty of Agriculture, University of Novi Sad, Trg D. Obradovica 8, 21000 Novi Sad, Serbia
}

Received: 6 Mar. 2013, Revised: 7 Jul. 2013, Accepted: 9 Jul. 2013

Published online: 1 Mar. 2014

\begin{abstract}
The paper discusses the problem of performing the prioritization of decision elements within the multicriteria optimization method, analytic hierarchy process (AHP), with incomplete information. An approach is proposed on how to fill in the gap in the pair-wise comparison matrix generated within an AHP standard procedure; that is, to reproduce one missing judgment of the decision maker while assuring the reproduced judgment belongs to the same ratio scale used while other judgments are elicited. The first-level transitivity rule (FLTR) approach is proposed based on screening matrix entries in the neighborhood of a missing one. Scaling (where necessary) and geometric averaging of screened entries allows filling of the gap in the matrix and later prioritization of involved decision elements by the eigenvector, or any other known method. Illustrative examples are provided to compare the proposed method with the other two known methods also aimed to fill-in gaps in AHP matrices. The results indicate some similarities in attaining consistency. However, unlike other methods, the FLTR assures coherency of the generating process in a sense that all numeric values in a matrix (original entries, plus one generated) come from the same ratio scale and have correct element-wise semantic equivalents.
\end{abstract}

Keywords: Analytic hierarchy process, comparison matrix, empty entries, transitivity

\section{Introduction}

One of the key issues in decision making is eliciting judgments from the decision maker (DM) about the importance of a given set of decision elements. If a problem can be structured hierarchically, then the fundamental Saaty's 9-point ratio scale in Table 1 [1] can serve as an efficient tool to assess this hierarchy by performing pair-wise comparisons. The analytic hierarchy process (AHP) [1], a well-known multicriteria optimization method, exploits this feature to create so-called local comparison matrices at all levels of a hierarchy. In standard AHP, an eigenvector (EV) method is used for deriving weights from local matrices; the EV is called the prioritization method, and the computational procedure is consequently called prioritization. After local weights are calculated at all levels of the hierarchy, a synthesis consists of multiplying the criterion-specific weight of the alternative with the corresponding criterion weight and summing up the results to obtain composite weights of the alternative with respect to the goal; this procedure is unique for all alternatives and all criteria.
Table 1: The fundamental Saaty's scale for the comparative judgments

\begin{tabular}{ll}
\hline Numerical value & Verbal terms \\
\hline 1 & Equally important \\
3 & Moderately more important \\
5 & Strongly more important \\
7 & Very strongly more important \\
9 & Extremely more important \\
$2,4,6,8$ & Intermediate values \\
\hline
\end{tabular}

If all comparisons are performed properly by the DM, then AHP synthesis is straightforward. However, if the DM for various reasons fails to make some judgments, then there are empty cells in the corresponding local matrices. The first case can be treated as decision making with complete information, and the other case with incomplete information. Harker [2] discussed three reasons why one would want to make fewer than the full set of judgments for each of one or more sets of factors in an AHP model:

\footnotetext{
*Corresponding author e-mail: bojans@polj.uns.ac.rs
} 
1.to reduce the time to make judgment

2.he/she is unwilling to make a direct comparison between two particular elements

3.he/she is unsure about some comparisons

After reviewing the literature, Ishizaka and Labib [3] state that there are three categories of papers related to incomplete matrices: calculation of missing comparisons, starting rules and stopping rules.

This paper deals with the calculation of missing comparisons. Different ways to tackle the missing judgments problem, both in individual and group decision-making contexts, can be found in the literature. Harker $[4,5]$, was one of the first who proposed how to solve the incomplete matrices problem. In [4], he takes the geometric average of all the indirectly calculated missing comparisons, based on the concept of connecting path.

Connecting paths in fact implement the general transitive rule (GTR) based on the following mathematics: if $a_{i j}(i \neq j)$ is missed in a positive reciprocal matrix, $a_{i j}$ can be determined by a connecting path of size $k, C P_{k}$, as follows:

$$
C P_{k}=a_{i, p 1} a_{p 1, p 2} \ldots a_{p k, j}
$$

The missing element $a_{i j}$ is a geometric mean of all connecting paths related to $a_{i j}$ :

$$
a_{i j}=\sqrt[q]{\prod_{k=1}^{q} C P_{k}}
$$

where $C P_{k}$ is a connecting path, $k$ defines the number of elements in the connecting path, and $q$ is the number of all possible connecting paths. A major drawback of this method is that the number of connecting paths can be high for large matrices. For example, for a matrix of size 10, the number of connecting paths is 109.000 .

Another approach is given in [5], where eigenvector is derived directly without estimating unknown comparisons.

Most recent methods used for solving problem of incomplete matrices are: consistency optimization [6]; the neural network based method [7,8]; connecting paths [9]; van Udens approximation rule for estimating missing judgment [10,11]; and graph representation [12], to name but a few.

Simulation employed in [7] comparing the efficiency of four methods (connecting paths, revised geometric mean method [2], characteristic polynomial-based method [13], and neural network based method), showed that connecting paths can be considered as the most efficient for small-sized matrices.

We propose an adapted version of GTR method for generating missing data in matrices where gaps occur. Only a case with one missing entry in a given matrix is considered, assuming that a reciprocal and symmetric entry (with respect to main diagonal) is also missing. A method is named the First-level transitive rule (FLTR) to indicate that the transitive property of pair-wise comparisons is considered, and only the first and explicit transitive relations between compared elements are used to perform the generation of missing data.

Two matrices of size 5 are used to illustrate an approach. Comparisons of FLTR's results are provided with respect to two other well known approaches in generating missing data in comparison matrices, Harker's [2] and van Uden's method [10]. Because the computed consistency indices (CR), the total Euclidean distances (ED) and minimum violations criterion (MV) usually serve as an indication of the decision-making quality, these three consistency measures are used in our comparisons. In order to create common framework, the prioritization is performed with the eigenvector $(\mathrm{EV})$ method in all cases, and a standard 9-point fundamental ratio scale [1] is used for eliciting judgments of decision elements. Based on our best experience with AHP, the results presented here and compared with the results obtained by other researchers, the FLTR is correct and a trustful concept which produces consistent results within the AHP philosophy based on its uniqueness (in application) of prioritization method, scale and consistency measurement. Moreover, proposed method is conceptually clear, robust in use for any size of comparison matrix, any level of its consistency, and any single missing entry; and finally, it is easy to implement FLTR in AHP-related computer software. Finally, FLTR is applicable if any other ratio scale is used, not only Saaty's, because its inherent property is normalization and preserving that reproduced missing data belong to the discrete set of values defined in the scale as associates to semantic statements used by the decision maker while eliciting his/her judgments.

\section{Analytic hierarchy process in brief}

\subsection{Main features}

AHP is a multicriteria method aimed at supporting decision-making processes in individual and group contexts. The core of AHP lies in presenting the problem as a hierarchy and comparing the hierarchical elements in a pair-wise manner using Saaty's 9-point scale to express the importance of one element over another in regards to the element in the higher level. The major feature of AHP is that it involves a variety of tangible and intangible goals, attributes, and other decision elements. In addition, it reduces complex decisions to a series of pair-wise comparisons; implements a structured, repeatable, and justifiable decision-making approach; and builds consensus.

\subsection{Pair-wise comparisons}

Without losing generality, the problem can be formalized by considering the prioritization of $n$ elements 
$E_{1}, E_{2}, \ldots, E_{n}$ at a given level of hierarchy. The DM semantically compares any two elements $E_{i}$ and $E_{j}$ and indirectly (verbally) or directly (numerically), using the scale in Table 1 , assigns the value $a_{i j}$ that represents a judgment of the relative importance of decision element $E_{i}$ over $E_{j}$. If element $E_{i}$ is of the same importance for the DM, then $a_{i j}=1$, and if $E_{i}$ is preferred to $E_{j}$, then $a_{i j}>1$. The reciprocal property $a_{j i}=1 / a_{i j}$ by assumption always holds, and $a_{i i}=1$ for all $i=1,2, \ldots, n$.

If $n$ elements of one level of hierarchy are compared regarding the element in the upper level, a comparison matrix $A$ has the following quadratic form:

$$
A=\left(\begin{array}{cccc}
a_{11} & a_{11} & \ldots & a_{1 n} \\
a_{21} & a_{22} & \ldots & a_{2 n} \\
\vdots & \vdots & \ddots & \vdots \\
a_{n 1} & a_{n 2} & \ldots & a_{n n}
\end{array}\right)
$$

Each matrix element $a_{i j}$ provided by the DM is a subjective judgment of the mutual importance of the two elements, $i$ and $j$. If the DM is fully consistent, then the transition rule $a_{i j} a_{j k}=a_{i k}$ should apply for all $i, j$, and $k$ in the range 1 to $n$.

The problem is to derive the priority vector $w=\left(w_{1}, w_{2}, \ldots, w_{n}\right)^{T}$ from the matrix, providing the elements of $w$ satisfy the relation:

$$
a_{i j}=w_{i} / w_{j}
$$

where $w_{i}$ and $w_{j}$ are the local weights of elements $i$ and $j$ regarding the element in the upper level. So, the weight's vector $w$ that corresponds to matrix (3) comprises the local weights of all the elements in the given hierarchy level regarding the element in the upper level.

However, vector $w$ is unknown, and the problem is that there is no such unique vector because of the well-known inconsistencies of the DM or the limitations imposed by any used scale. In order to measure the successfulness of the $w$ vector determination by any of the existing methods, e.g. $[1,14,15,16,17,18,19,20,21]$, one can define several metrics and compare the original matrix $A$ and corresponding matrix $C$ :

$$
C=\left(\begin{array}{cccc}
w_{1} / w_{1} & w_{1} / w_{2} & \ldots & w_{1} / w_{n} \\
w_{2} / w_{1} & w_{2} / w_{2} & \ldots & w_{2} / w_{n} \\
\vdots & \vdots & \ddots & \vdots \\
w_{n} / w_{1} & w_{n} / w_{2} & \ldots & w_{n} / w_{n}
\end{array}\right)
$$

An issue of comparisons is usually considered as 'measuring consistency', and several measures have been defined by different authors as will be discussed in section 2.4 .

\subsection{Prioritization by the Eigenvector method}

Over the years, several methods have been proposed for estimating the weights from a matrix of pairwise comparisons, including additive normalization (AN), eigenvector (EV), logarithmic least squares (LLS), weighted logarithmic least square (WLS), logarithmic goal programing (LGP), fuzzy preference programing (FPP), and others. A brief description of these competing methods is provided by Harker and Vargas [4], and Srdjevic [20]. Herein, we present the main features of the EV method that is commonly used in practice, and which we used in our research.

The EV method is a widely used method to generate priority vectors for given pairwise matrices. The method, originally proposed by Saaty [1], solves an eigenvalue problem associated with a quadratic pairwise comparison matrix of size $n$ and is nicely described in Chandran et al [22] as partly quoted below with some additions for better understanding.

Let $A=\left(a_{i j}\right)$, for $i, j=1,2, \ldots, n$ denote a square pairwise comparison matrix, where entry $a_{i j}$ gives the importance of element $i$ relative to element $j$. Each entry is a positive value $\left(a_{i j}>0\right)$ with a reciprocal $a_{j i}=1 / a_{i j}$ (for all $i, j=1,2, \ldots, n$ ). The decision maker wants to compute a vector of weights $\left(w_{1}, w_{2}, \ldots, w_{n}\right)$ associated with $A$.

If the matrix $A$ is consistent (that is, $a_{i j}=a_{i k} a_{k j}$ for all $i, j, k=1,2, \ldots, n)$, then $A$ contains no errors. Therefore, the weights are already known, and we have

$$
a_{i j}=w_{i} / w_{j}, \quad i, j=1,2, \ldots, n .
$$

Summing over all $j$, we obtain

$$
\sum_{j=1}^{n} a_{i j} w_{j}=n w_{i}, \quad i, j=1,2, \ldots, n .
$$

In matrix notation, it is equivalent to

$$
A w=n w, \quad e^{T} w=1 .
$$

The vector $w$ is the principal right eigenvector of matrix $A$ corresponding to the eigenvalue $n$. If the vector of weights is not known, then it can be estimated from the pairwise comparison of matrix $A^{\prime}$ generated by the decision maker and solving

$$
A^{\prime} w^{\prime}=\lambda^{\prime} w^{\prime}, \quad e^{T} w^{\prime}=1
$$

for $w^{\prime}$. The matrix $A^{\prime}$ contains the pairwise judgments of the decision maker and approximates the matrix $A$ whose entries are unknown. In Eq. (9), $\lambda^{\prime}$ is an eigenvalue of $A^{\prime}$, and $w^{\prime}$ is the estimated vector of weights. Saaty [1] uses the largest eigenvalue $\lambda_{\max }$ of $A^{\prime}$ when solving for $w$ in

$$
A^{\prime} w^{\prime}=\lambda_{\max } w^{\prime}, \quad e^{T} w^{\prime}=1 .
$$

Saaty has shown that $\lambda_{\max }$ is always greater than or equal to $n$ and if its value is close to $n$, then the estimated vector of weights $w^{\prime}$ solves Eq. (10) approximately.

A good estimate of the principal eigenvector for an inconsistent matrix is obtainable by consecutively 
squaring the matrix, normalizing the row sums each time, and stopping the procedure when the difference between the normalized sums in two consecutive calculations is smaller than a prescribed value.

It was shown by various researchers that for small deviations around the consistent ratios $w_{i} / w_{j}$, the EV method gives a reasonably good approximation of the priorities' vector. However, when the inconsistencies are large, it is generally accepted that the solutions are not so satisfactory.

\subsection{Consistency measures}

Consistency analysis of the individual DM can be based on the $C R$ defined by Saaty [1], and the total $L^{2} E D$ for each comparison matrix. Whichever method is used to derive the priority vector from the given local AHP matrix [20], whether it already has all the entries elicited from the DM, or is filled in with FLTR data, consistency is necessary to measure in order to preserve integrity that the outcomes are trustful.

Standard AHP uses EV, the prioritization method, and the consistency coefficient $C R$ to indicate the inconsistency of the DM [1]. The other commonly used consistency measures are the total Euclidean distance ED and minimum violation (rank reversal) criterion $M V$.

\subsubsection{Consistency ratio}

The $C R$ is calculated as a part of the standard AHP procedure. First, the consistency index $(C I)$ is calculated using the following equation:

$$
C I=\frac{\lambda_{\max }-n}{n-1}
$$

where $\lambda_{\max }$ is the principal eigenvalue of matrix (3). Knowing the consistency index and random consistency index $(R I)$ defined also by Saaty [1], the consistency ratio is obtained:

$$
C R=\frac{C I}{R I} \text {. }
$$

Saaty [1] suggested considering the maximum level of the DM's inconsistency to be 0.10 ; that is, $C R$ should be less or equal to 0.10 .

\subsubsection{Total $L^{2}$ Euclidean distance}

Total $L^{2}$ Euclidean distance

$$
E D=\left[\sum_{i=1}^{n} \sum_{j=1}^{n}\left(a_{i j}-w_{i} / w_{j}\right)^{2}\right]^{1 / 2}
$$

represents the distance measured between all elements in a comparison matrix (3) and the corresponding elements in the related matrix (5). The lower is the $E D$, and the higher is the consistency of the DM.

\subsubsection{Minimum violations $(M V)$}

This measure (criterion) is given by Eqs. (14) and (15):

$$
\begin{gathered}
M V=\frac{1}{n^{2}} \sum_{i=1}^{n} \sum_{j=1}^{n} L_{i j} \\
L_{i j}= \begin{cases}1 & \text { if } w_{i}>w_{j} \text { and } a_{j i}>1 \\
0.5 & \text { if } w_{i}=w_{j} \text { and } a_{j i} \neq 1 \\
0.5 & \text { if } w_{i} \neq w_{j} \text { and } a_{j i}=1 \\
0 & \text { otherwise } .\end{cases}
\end{gathered}
$$

Eqs. (14) and (15) sum up and then average all violations associated with the priority vector $w$. The conditions of violation defined by (15) penalize possible order reversals such as this: if the $j$ th alternative is preferred to the $i$ th one (i.e. $a_{j i}>1$ ), but the derived priorities are such that $w_{i}>w_{j}$, then there is a violation, or element preference reversal (14).

\section{Two well-known methods for filling-in incomplete matrices}

\subsection{Harker's method [4]}

For given incomplete matrix $A\left(a_{i j}\right)$, corresponding reciprocal matrix $\widetilde{A}\left(\widetilde{a_{i j}}\right)$ is defined as

$$
\widetilde{a_{i j}}=\left\{\begin{array}{lrl}
1+m_{i} & \text { if } \quad i=j \\
0 & \text { if } & a_{i j} \text { is missing } \\
a_{i j} & \text { otherwise }
\end{array}\right.
$$

where $m_{i}$ is the number of missing elements in the $i$-th row.

\subsection{Van Uden's method [10]}

If only one entry $a_{i k}$ is missing, van Uden proposes the following equation for calculating the missing element

$$
a_{i k}=\sqrt[n-2]{\frac{X}{Y}}
$$

where $X=\prod_{j \neq k}^{j} a_{i j}$ and $Y=\prod_{j \neq i}^{j} a_{k j}$. Also, $a_{i k}=\frac{w_{i}}{w_{k}}$, assuming that matrix $W$ is a consistent approximation of matrix $A$.

\section{First-level transitive rule (FLTR) method}

In pertinent literature, it is commonly said that a given square comparison matrix $A=\left\{a_{i j}\right\}$ of order $\mathrm{n}$ is fully consistent if the transitive rule $a_{i j}=a_{i k} \cdot a_{k j}$ applies for all $i, j$, and $k$. In reality, one may expect only matrices of an order up to 4 to be fully consistent. In most cases, there 
are one or more violations of the transition rule and inconsistency occurs.

The general transitive rule (GTR) presented in the previous section deals with numerous connecting paths; that is, with chains of all matrix entries with equal inner (neighbor) indices. For large matrices, the general rule is not efficient.

If only FLTR is applied, then in the case of say a matrix of order five and missing entry $a_{45}$, there are only three first-level transitions, namely:

$$
\begin{aligned}
& a_{45}=a_{41} \cdot a_{15} \\
& a_{45}=a_{42} \cdot a_{25} \\
& a_{45}=a_{43} \cdot a_{35}
\end{aligned}
$$

The two remaining first-level transitions

$$
\begin{aligned}
& a_{45}=a_{44} \cdot a_{45} \\
& a_{45}=a_{45} \cdot a_{55}
\end{aligned}
$$

make no sense, because $a_{44}=a_{55}=1$.

If a matrix is of order seven, then in the case of, for example, element $a_{57}$, FLTR gives five transitions:

$$
\begin{aligned}
& a_{57}=a_{51} \cdot a_{17} \\
& a_{57}=a_{52} \cdot a_{27} \\
& a_{57}=a_{53} \cdot a_{37} \\
& a_{57}=a_{54} \cdot a_{47} \\
& a_{57}=a_{56} \cdot a_{67}
\end{aligned}
$$

Again, two remaining FLTR transitions

$$
\begin{aligned}
& a_{57}=a_{55} \cdot a_{57} \\
& a_{57}=a_{57} \cdot a_{77}
\end{aligned}
$$

do not provide any relevant information, because $a_{55}=$ $a_{77}=1$.

Obviously, in the first step FLTR handles only a reasonable number of direct transition values, computed for a missing matrix entry $a_{i k}$ (and also reciprocal and symmetrical $a_{k i}=1 / a_{i k}$ ) based on existing entries in the same matrix. For matrix of order $n$, inner products of existing entries is $n-2$, as also implied by formulas given in (10).

The second, final step in applying FLTR is to compute the missing entry from the computed transitive values. There are several possible ways to do that. We propose to geometrically average and round the result to the closest numerical value from Saaty's scale. If at least one transition value is out of Saaty's scale range $\{1-9\}$, which may occur quite often, then the computed transition values should be scaled to fall within range before proceeding with geometric averaging and rounding. This is methodologically correct because it preserves the eliciting of judgments from the decision maker is virtually done by fully respecting the scale during the complete judgment process, without exemptions as implicitly permitted in other two well known methods $[2,10]$.

Experiments with different matrices indicate that FLTR is superior to the general transition rule (GTR) in both the quality of generating missing data, and programming and computation requirements. Saaty's scale can be considered as a discrete set of 17 semantic statements with associated numerical values as given in Table 2. For simplicity, let the scale be represented as a discrete set $S$ with elements: $s_{1}=1, s_{2}=2, \ldots, s_{9}=9$, $s_{10}=1 / 2, s_{11}=1 / 3, \ldots, s_{17}=1 / 9$. Let us call these numerical values as 'scale points', and the distance between largest $\{9\}$ and lowest value $\{1 / 9\}$, as the scale range $R=9-1 / 9=80 / 9 \approx 8.89$.

The statements from the semantic part of the Saaty's scale can be considered as equally distanced. In such a discrete domain, it can be adopted analogously that their numerical associates are also equally distanced; that is the distance between points $s_{10}$ and $s_{11}(1 / 2$ and $1 / 3)$ is considered the same as between points $s_{11}$ and $s_{12}$, regardless of their real numerical distances as it comes from only numerical part of the Saaty's scale. In this way we create a base for unique treatment of values contained in the comparison matrix, both those that exist and the one which is missing.

Assume now that in given comparison matrix $A$ of size $n$, there is a missing value $a_{i k}$. Consequently, the element $a_{k i}$ is also missing because matrix $A$ is by assumption reciprocal. Once one of those two missing elements is derived, the other is simple to compute, e.g. $a_{k i}=1 / a_{i k}$.

First order transition rule defines a set of inner products

$X=\left\{\left(a_{i j} a_{j k}\right)^{l}: \quad i \neq j ; \quad j \neq k ; \quad i, j, k=1,2, \ldots, n ; \quad l=1,2, \ldots m\right\}$

A set $X$ consists of exactly $m=n-2$ different inner products of existing elements. There are two possible cases:

\section{Case 1:}

If all products in $X$ set by an individual value fall within the range $\{1 / 9-9\}$ in Saaty's scale, and this range encapsulates the discrete points of $S$, then the approximation of missing element $a_{i k}$ is computed as a geometric average

$$
a_{i k}^{\prime}=\left[\prod_{l=1}^{m}\left(a_{i j} a_{j k}\right)^{l}\right]^{1 / m}
$$

This value falls into the range $\{1 / 9-9\}$ and it only remains to round the value to the nearest discrete scale point from the set $S$. This point is then declared as FLTR generated missing value $a_{i k}$ from the matrix $A$. 


\section{Case 2:}

If at least one of the inner products in $X$ violates the range $\{1 / 9,9\}$ in Saaty's scale, all inner products have to be additively normalized and then scaled to fall within the scale. For a 'distance' $R$ between the upper and lower limit of the scale, normalization and scaling give

$$
\left(a_{i j} a_{j k}\right)^{l(s)}=\left[\left(a_{i j} a_{j k}\right)^{l} / \sum_{l=1}^{m}\left(a_{i j} a_{j k}\right)^{l}\right] \cdot R, \quad l=1, \ldots, m .
$$

Geometric averaging of scaled values

$$
a_{i k}^{\prime}=\left[\prod_{l=1}^{m}\left(a_{i j} a_{j k}\right)^{l(s)}\right]^{1 / m}
$$

produces value which falls within the range $R$. Simply matching the nearest discrete value on the scale gives the required missing element $a_{i k}$.

\section{Numerical examples}

Two complete comparison matrices $A 1_{\text {complete }}$ and $A 2_{\text {complete }}$ are taken from [23] as obtained from real decision maker (DM), an expert in agricultural irrigation. The decision maker used Saaty's scale (Table 1) to judge five decision elements at a given level of the hierarchy by comparing them in pairs versus two different decision elements in the upper level (matrices (22) and (23)).

$$
\begin{aligned}
A 1_{\text {complete }} & =\left(\begin{array}{ccccc}
1 & 1 / 4 & 1 / 5 & 1 / 3 & 1 / 2 \\
4 & 1 & 1 / 3 & 1 / 2 & 1 / 3 \\
5 & 3 & 1 & 2 & 3 \\
3 & 2 & 1 / 2 & 1 & 3 \\
2 & 3 & 1 / 3 & 1 / 3 & 1
\end{array}\right) \\
A 2_{\text {complete }} & =\left(\begin{array}{ccccc}
1 & 4 & 2 & 4 & 5 \\
1 / 4 & 1 & 1 & 2 & 4 \\
1 / 2 & 1 & 1 & 3 & 3 \\
1 / 4 & 1 / 2 & 1 / 3 & 1 & 5 \\
1 / 5 & 1 / 4 & 1 / 3 & 1 / 5 & 1
\end{array}\right)
\end{aligned}
$$

EV prioritization produced priority vectors presented in Table 2 along with computed consistency measures according to Eqs. (9-13).

Table 2: The AHP results (complete matrices)

\begin{tabular}{ccccccccc}
\hline & \multicolumn{4}{c}{ Priority vector } & \multicolumn{4}{c}{ Consistency measures } \\
Matrix & $w_{1}$ & $w_{2}$ & $w_{3}$ & $w_{4}$ & $w_{5}$ & $C R$ & $E D$ & $M V$ \\
\hline \hline$A 1_{\text {complete }}$ & 0.063 & 0.128 & 0.387 & 0.257 & 0.165 & 0.091 & 3.580 & 0.00 \\
$A 2_{\text {complete }}$ & 0.435 & 0.178 & 0.212 & 0.122 & 0.052 & 0.075 & 4.938 & $1.00^{*}$ \\
\hline \multicolumn{4}{c}{ Violation of 0.5 is recorded at elements $a_{23}$ and $a_{32}$ in matrix $A 2$}
\end{tabular}

Assume now that the DM, by chance or intentionally, missed comparing some elements in matrices $A 1_{\text {complete }}$ and $A 2_{\text {complete }}$; that is, let the two missing judgments be $a_{24}$ and $a_{15}$, respectively. By assumption, their reciprocals are also missing symmetrically with respect to main diagonal in both matrices, $A 1_{\text {missing }}$ (24) and $A 2_{\text {missing }}$ (25). Missing entries have to be computed preserving that consistency measures $C R, E D$ and $M V$ are forced to be minimal.

$$
\begin{aligned}
A 1_{\text {missing }} & =\left(\begin{array}{ccccc}
1 & 1 / 4 & 1 / 5 & 1 / 3 & 1 / 2 \\
4 & 1 & 1 / 3 & --^{*} & 1 / 3 \\
5 & 3 & 1 & 2 & 3 \\
3 & --^{*} & 1 / 2 & 1 & 3 \\
2 & 3 & 1 / 3 & 1 / 3 & 1
\end{array}\right) \\
A 2_{\text {missing }} & =\left(\begin{array}{ccccc}
1 & 4 & 2 & 4 & --^{*} \\
1 / 4 & 1 & 1 & 2 & 4 \\
1 / 2 & 1 & 1 & 3 & 3 \\
1 / 4 & 1 / 2 & 1 / 3 & 1 & 5 \\
--{ }^{*} & 1 / 4 & 1 / 3 & 1 / 5 & 1
\end{array}\right)
\end{aligned}
$$

\section{Reproducing missing judgments by the Harker's method}

Application of the Harker's method gives priority vectors and consistency values $C R, E D$ and $M V$ as shown in Table 3. Based on computed weights, the missing entries in two matrices are simple to obtain via formula $a_{i j}=w_{i} / w_{j}$, and correspondingly $a_{j i}=w_{j} / w_{i}$, (matrices $A 1_{\text {Harker }}$ and $A 2_{\text {Harker }}$ ). Notice that reproduced values do not match Saaty's scale by not precisely corresponding to any linguistic term related to judging mutual importance of two given (compared) elements. Moreover, the computed value $a_{15}=11.5$ in (27) is out of scale, the remedy to which is to set $a_{15}=9$ to match end point of the scale which says that element 1 is absolutely dominant to element 5; however, this shift does not come from the Harker's method in any sense, and is therefore unjustified.

Notice that consistency measures for matrix $A 2_{\text {Harker }}$ in Table 3 are better than corresponding values given in Table 2 for original (full) matrix. This result is expected because Harker's method is constructed to produce it. However, as said before, it is not justified in a semantic sense. For the sake of completeness, in case of matrix $A 1$ Harker's method almost exactly reproduced original entry $a_{24}$, and in turn it's reciprocal $a_{42}$ (Cf. matrices (26) and (22)).

Table 3: The AHP results (Harker's method)

\begin{tabular}{ccccccccc}
\hline & \multicolumn{4}{c}{ Priority vector } & \multicolumn{4}{c}{ Consistency measures } \\
Matrix & $w_{1}$ & $w_{2}$ & $w_{3}$ & $w_{4}$ & $w_{5}$ & $C R$ & $E D$ & $M V$ \\
\hline \hline$A 1_{\text {Harker }}$ & 0.063 & 0.128 & 0.387 & 0.257 & 0.165 & 0.091 & 3.580 & 0.00 \\
$A 2_{\text {Harker }}$ & 0.474 & 0.168 & 0.205 & 0.112 & 0.041 & 0.052 & 3.530 & $1.00^{*}$ \\
\hline *Violation of 0.5 is recorded at elements $a_{23}$ and $a_{32}$ in matrix $A 2$
\end{tabular}




$$
\begin{gathered}
A 1_{\text {Harker }}=\left(\begin{array}{ccccc}
1 & 1 / 4 & 1 / 5 & 1 / 3 & 1 / 2 \\
4 & 1 & 1 / 3 & \mathbf{0 . 5 0}^{*} & 1 / 3 \\
5 & 3 & 1 & 2 & 3 \\
3 & \mathbf{2 . 0 1}^{*} & 1 / 2 & 1 & 3 \\
2 & 3 & 1 / 3 & 1 / 3 & 1
\end{array}\right) \\
A 2_{\text {Harker }}=\left(\begin{array}{ccccc}
1 & 4 & 2 & 4 & \mathbf{1 1 . 5}^{*} \\
1 / 4 & 1 & 1 & 2 & 4 \\
1 / 2 & 1 & 1 & 3 & 3 \\
1 / 4 & 1 / 2 & 1 / 3 & 1 & 5 \\
\mathbf{0 . 0 9}^{*} & 1 / 4 & 1 / 3 & 1 / 5 & 1
\end{array}\right)
\end{gathered}
$$

\section{Reproducing missing judgments by the van Uden's method}

The Van Uden's method produces priority vectors and consistency values for matrices $A 1$ and $A 2$ similar to those produced by the Harker's method, Table 4. Unlike the Harker's method, computing the missing entries is direct. Missing values, as well as their reciprocals, are presented in (28) and (29). Notice that, again, all reproduced values do not match Saaty's scale. In matrix $A 2_{v U d e n}$ computed value $a_{15}=12.4$ is out of scale, even further than in previous case.

The consistency measures for matrix $A 2_{v U d e n}$ in Table 4 are better than corresponding values given in Table 2 . This result is also expected but, from the same argumentation as before, not justified in a semantic sense. In the case of matrix $A 1$, van Uden's method reproduced an acceptable value for original entry $a_{24}$ and its reciprocal $a_{42}$ (Cf. matrices (28) and (22)).

Table 4: The AHP results (Van Uden's method)

\begin{tabular}{ccccccccc}
\hline & \multicolumn{4}{c}{ Priority vector } & \multicolumn{4}{c}{ Consistency measures } \\
Matrix & $w_{1}$ & $w_{2}$ & $w_{3}$ & $w_{4}$ & $w_{5}$ & $C R$ & $E D$ & $M V$ \\
\hline \hline$A 1_{\text {vUden }}$ & 0.063 & 0.126 & 0.387 & 0.260 & 0.163 & 0.090 & 3.592 & 0.00 \\
$A 2_{\text {vUden }}$ & 0.474 & 0.168 & 0.205 & 0.112 & 0.041 & 0.052 & 3.589 & $1.00^{*}$ \\
\hline
\end{tabular}

$$
\begin{aligned}
A 1_{v U \text { den }} & =\left(\begin{array}{ccccc}
1 & 1 / 4 & 1 / 5 & 1 / 3 & 1 / 2 \\
4 & 1 & 1 / 3 & \mathbf{0 . 4 6} & 1 / 3 \\
5 & 3 & 1 & 2 & 3 \\
3 & \mathbf{2 . 1 6}^{*} & 1 / 2 & 1 & 3 \\
2 & 3 & 1 / 3 & 1 / 3 & 1
\end{array}\right) \\
A 2_{v \text { Uden }} & =\left(\begin{array}{ccccc}
1 & 4 & 2 & 4 & \mathbf{1 2 . 4}^{*} \\
1 / 4 & 1 & 1 & 2 & 4 \\
1 / 2 & 1 & 1 & 3 & 3 \\
1 / 4 & 1 / 2 & 1 / 3 & 1 & 5 \\
\mathbf{0 . 0 8}^{*} & 1 / 4 & 1 / 3 & 1 / 5 & 1
\end{array}\right)
\end{aligned}
$$

\section{Reproducing missing judgments by the FLTR method}

Following the first-level transitive rule approach, the missing judgments are computed as follows:

Matrix A1: missing element is $a_{24}$

$$
\begin{aligned}
& a_{24}{ }^{1}=a_{21} \cdot a_{14}=4 \cdot(1 / 3)=4 / 3 \\
& a_{24}{ }^{2}=a_{23} \cdot a_{34}=(1 / 3) \cdot 2=2 / 3 \\
& a_{24}{ }^{3}=a_{25} \cdot a_{54}=(1 / 3) \cdot(1 / 3)=1 / 9
\end{aligned}
$$

Because all inner products are within the range $\{1 / 9-9\}$ in Saaty's scale, Case 1 applies and Eq. 19 gives

$$
a_{24}^{\prime}=\sqrt[3]{a_{24}{ }^{1} a_{24}^{2} a_{24}^{3}} \approx 0.462 .
$$

The missing entry (judgment) is $a_{24}=1 / 2$ because it is the closest value in the scale $S$. Symmetric entry is $a_{42}=2$.

Matrix A2: missing element is $a_{15}$

$$
\begin{aligned}
& a_{15}{ }^{1}=a_{12} \cdot a_{25}=4 \cdot 4=16 \\
& a_{15}{ }^{2}=a_{13} \cdot a_{35}=2 \cdot 3=6 \\
& a_{15}{ }^{3}=a_{14} \cdot a_{45}=4 \cdot 5=20
\end{aligned}
$$

Because two of the computed values violate the range $\{1 / 9,9\}$ in Saaty's scale, Case 2 applies and all values have to be additively normalized and scaled to fall within the scale range. Application of Eq. (20) gives

$$
\begin{array}{ll}
a_{15}{ }^{1(n)}=16 / 42=0.38, & a_{15^{1(s)}}=0.38 \cdot(80 / 9)=3.38 \\
a_{15}{ }^{2(n)}=6 / 42=0.38, & a_{15^{2(s)}}=0.14 \cdot(80 / 9)=1.24 \\
a_{15}{ }^{3(n)}=20 / 42=0.48, & a_{15}{ }^{3(s)}=0.48 \cdot(80 / 9)=4.27
\end{array}
$$

Therefore, the missing entry (judgment) is $a_{15}=3$. Symmetric entry is $a_{51}=1 / 3$.

With these two values inserted into the matrices in (30) and (31), it is possible to perform EV prioritization for both matrices and to derive the final weights of all compared elements. Table 5 contains the computed weights, and consistency measures $C R, E D$ and $M V$. In case of matrix $A 2_{f l t r}$, value $a_{15}=3$, as generated by the FLTR method, belongs to the Saaty's scale with associated semantic value 'decision element 1 is moderately more important than decision element 5;' recall that the original (true) value is $a_{15}=5$ which corresponds to the judgment: 'decision element 1 is strongly more important than decision element 5.' These two statements do not differ significantly and the 
computed priority vector with the FLTR method has an absolute value closer to the original priority vector than the vectors obtained by other two methods (0.046 vs. 0.077 , in favor of FLTR). On the other side, in case of FLTR method, the consistency measures $C R$ and $E D$ are slightly worse than those obtained by Harker's and van Uden's method. Even so, they are methodologically justified while Harker's and van Uden's are not because generated values do not belong to the scale which is already used for the remaining part of matrix $A 2$.

In the case of matrix $A 1$, the FLTR method exactly reproduced original value $a_{24}$ and it's reciprocal. Notice that base assumption in applying FLTR method is that the final (generated missing) values must belong to the used scale (in this case Saaty's scale).

Table 5: The AHP results (FLTR method)

\begin{tabular}{ccccccccc}
\hline & \multicolumn{4}{c}{ Priority vector } & \multicolumn{4}{c}{ Consistency measures } \\
Matrix & $w_{1}$ & $w_{2}$ & $w_{3}$ & $w_{4}$ & $w_{5}$ & $C R$ & $E D$ & $M V$ \\
\hline \hline$A 1_{\text {fltr }}$ & 0.063 & 0.128 & 0.383 & 0.257 & 0.165 & 0.091 & 3.580 & 0.00 \\
$A 2_{\text {fltr }}$ & 0.413 & 0.183 & 0.214 & 0.128 & 0.062 & 0.112 & 5.407 & $1.00^{*}$ \\
\hline${ }^{*}$ Violation of 0.5 is recorded at elements $a_{23}$ and $a_{32}$ in matrix $A 2$
\end{tabular}

$$
\begin{aligned}
A 1_{\text {fltr }} & =\left(\begin{array}{ccccc}
1 & 1 / 4 & 1 / 5 & 1 / 3 & 1 / 2 \\
4 & 1 & 1 / 3 & \mathbf{1} \mathbf{2}^{*} & 1 / 3 \\
5 & 3 & 1 & 2 & 3 \\
3 & \mathbf{2}^{*} & 1 / 2 & 1 & 3 \\
2 & 3 & 1 / 3 & 1 / 3 & 1
\end{array}\right) \\
A 2_{\text {fltr }} & =\left(\begin{array}{ccccc}
1 & 4 & 2 & 4 & \mathbf{3}^{*} \\
1 / 4 & 1 & 1 & 2 & 4 \\
1 / 2 & 1 & 1 & 3 & 3 \\
1 / 4 & 1 / 2 & 1 / 3 & 1 & 5 \\
\mathbf{1 / 3} & 1 / 4 & 1 / 3 & 1 / 5 & 1
\end{array}\right)
\end{aligned}
$$

Notice that in matrix $A 1_{\text {complete }}(22)$ the DM provided the same judgment $a_{24}=1 / 2$ as the one generated by the FLTR. The judgment $a_{15}=5$, provided by the DM in matrix $A 2_{\text {complete }}(23)$, is slightly different from the one generated by the FLTR $\left(a_{15}=3\right)$.

For the sake of completeness, the decision maker has been asked to reconsider both matrices but with emptied positions $a_{24}\left(a_{42}\right)$ and $a_{15}\left(a_{51}\right)$. He spent some time reconsidering the whole problem and his previous judgments. Interesting to note, he exactly repeated the original judgments as given in (22) and (23).

It should be noted that an opposite situation could occur if the decision maker makes inconsistent judgments instead of obviously correct ones. More sensitivity analysis could possibly be necessary for such cases. In this particular case the decision maker was not asked to change his judgments in any way, and this sensitivity option will remain for future research.

Although the applied FLTR computation is just a mathematical procedure a posteriori, or a deductive operation independent of any further re-thinking about the problem or logical assessment of the judgment already made by the DM, the same claim is valid for other known methods for filling-in empty matrices. There are no foreseen better solutions in this regard.

\section{Conclusions}

In real-life decision making, it may occur that for various reasons the DM is unable or unwilling to judge all criteria vs. goal, or all alternatives vs. all criteria. This situation is known as decision making with incomplete information. In this paper, we discuss the problem within the AHP framework and propose a method to fill in the gaps in the pair-wise comparison matrices generated through elicitation of the DM's semantic judgments. A method for generating a numerical equivalent to the missing semantic judgment is named FLTR, after so-called first level transition rule; that is, proposed method exploits information from positions in a comparison matrix, neighbor to a position where the judgment is missing. Differently from some other known methods, FLTR assumes that generation of the missing judgment is achieved in a way that preserves the final result's belonging to the same scale which is used by the decision maker for eliciting his/her other judgments during standard AHP application. Therefore, FLTR involves geometric averaging and scaling of inner products that realize the transition rule, and the final matching of computed numerical to the scale used for all other pair-wise comparisons of decision elements in a hierarchy.

The proposed method can generate only one missing judgment in any local matrix and in turn enables prioritization for a given matrix, as well as the final AHP synthesis. FLTR method is applicable for any ratio scale and is independent of the prioritization method used. In later case the only restriction could be an issue of measuring consistency which is a problem by itself and is out of scope in this paper. Worthy to mention is that there are controversies among researchers about an issue of consistency; it could be a reason why a relatively 'small portion' of published papers related to AHP deal with consistency, both at individual and group level. In this work we also intentionally did not insist on consistency measures; some are just presented for comparison reasons.

Two tolerantly consistent matrices are used as a proof for concept examples, and FLTR is applied together with two other well-known methods. For comparison purposes common metrics in measuring consistency are used. The compared methods do not outperform the others and results are considered as competitive. However, our argument is that the offered FLTR approach is more coherent because generated missing data in comparison matrices can always have associated exact semantic statements, unlike in the other methods used for comparison. The obtained results we consider as 
promising, with anticipated positive outcomes in future research in solving group decision-making problems with incomplete information, e.g. cross-generation of missing data in matrices taken from different decision makers.

\section{Acknowledgement}

This work was supported in part by Serbian Ministry of Education, Science and Terchnological Development, and by Secretariat for Science and Technological Development of Vojvodina Province.

\section{References}

[1] T. L Saaty, The analytic hierarchy process, McGraw-Hill, New York, (1980).

[2] P. T Harker, Mathematic Modeling, 9, 353-360 (1980).

[3] A. Ishizaka and A. Labib, Expert Systems with Applications, 38, 14336-14345 (2011).

[4] P. T Harker and L. Vargas, Management Science, 33, 13831403 (1987).

[5] P. T Harker, Mathematical Modelling, 9, 837-848 (1987).

[6] M. Fedrizzi and S. Giove, European Journal of Operational Research, 183, 303-313 (2007).

[7] Y. C Hu and J. F Tsai, Applied Mathematics and Computation, 180, 53-62 (2006).

[8] J. A Gomez-Ruiz, M. Karanik, J.I Pelaez, Applied Mathematics and Computation, 216, 2959-2975 (2010).

[9] Q. Chen and E. Triantaphyllou, Estimating data for multicriteria decision making problems: Optimization techniques, Texts in Encyclopedia of Optimization, Kluwer, Boston, 2, 27-36 (2011).

[10] E. Van Uden, Estimating missing data in pairwise comparison matrices, Texts in Operational and Systems Research in the Face to Challenge the XXI Century, Methods and Techniques in Information Analysis and Decision Making, Academic Printing House, Warsaw, (2002).

[11] M. Kwiesielewicz and E. Van Uden, Ranking decision variants by subjective paired comparisons in cases with incomplete data, Texts in ICCSA 2003, LNCS, SpringerVerlag, 2669, 208-215 (2003).

[12] S. Bozoki, J. Fulop, J. Ronyai, Mathematical and Computer Modelling, 52, 318-333 (2010).

[13] S. Shiraishi, T. Obata, M. Daigo, Journal of Operational Research Society of Japan, 41, 404-414 (1998).

[14] G. Crawford and C. Williams, Journal of Mathematical Psychology, 29, 387-405 (1985).

[15] F. Zahedi, Socio-Economic Planning Science, 6, 347-354 (1986).

[16] E. Takeda, K. Cogger, P.L Yu, European Journal of Operational Research, 29, 360-369 (1987).

[17] V. N Kumar and L. Ganesh, European Journal of Operational Research, 95, 656-662 (1996).

[18] J. Barzilai, Journal of Operational Research Society, 48, 1226-1232 (1997).

[19] L. Mikhailov, Journal of Operational Research Society, 51, 341-349 (2000).
[20] B. Srdjevic, Computers and Operations Research, 32, 18971919 (2005).

[21] B. Srdjevic and Z. Srdjevic, Applied Mathematics and Computation, 218, 1254-1266 (2011).

[22] B. Chandran, B. Golden, E. Wasil, Computers and Operations Research, 32, 2235-2254 (2005).

[23] Z. Srdjevic, B. Srdjevic, S. Potkonjak, T. Zoranovic, Allocation of land to agricultural crops in presence of irrigation and drainage: an approach based on the analytic hierarchy process, Texts in Proceedings Melioration and Agriculture, Faculty of Agriculture, Novi Sad, Serbia and Montenegro, /in Serbian/, 222-239 (2002).

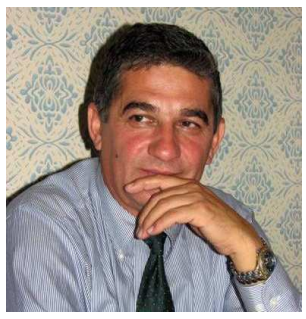

Bojan Srdjevic is a Full Professor in Water Resources Systems Analysis and Informatics. $\mathrm{He}$ is lecturing on various graduate and postgraduate courses at the Universities of Novi Sad (Serbia), Salvador (Brazil) and Stuttgart (Germany). His research interests include decision-making methodologies and supporting tools, and water resources planning and management. $\mathrm{He}$ is the author of more than 200 papers in international and national journals and conference proceedings, and a referee for IEEE, Elsevier, Springer, ASCE, IWA international journals, beside others.

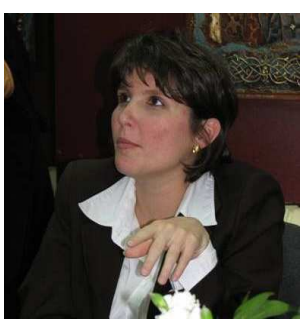

Zorica Srdjevic is an Associate Professor at the University of Novi Sad, Serbia. She is lecturing on various undergraduate, master and doctoral courses. Her research interests include multi-criteria analysis and decision-making methodologies and supporting tools. She is the author of more than 100 articles and other publications, mainly in peer-reviewed international and national journals. She is a referee for several international journals, including Elsevier and Springer.

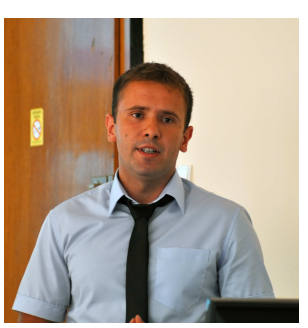

Bosko Blagojevic is a Ph.D. candidate working as a Teaching Assistant in Water Resources Systems Analysis and Informatics. His research focus is on development and application of decision-making methods in agriculture and water resources. He is the author of more than 30 papers in international and national journals and conference proceedings. 